\title{
Presentes y futuros: inercias y desafíos. Gestión y políticas culturales de cara al futuro ${ }^{1}$
}

\author{
Present and future: inertias and challenges. \\ Management and cultural policies facing the future
}

\section{Hugo Achugar**}

\footnotetext{
** CURE-UDELAR. Profesor Titular responsable del PDU Políticas Culturales y Políticas de la Cultura. $\bowtie$ hugoach@gmail.com
}

\begin{abstract}
Resumen
Ante el inexorable avance y desarrollo de las transformaciones e innovaciones tecnológicas parece que ha llegado el tiempo de preguntarse si nos estamos preparando para encarar los desafíos que estas presentan a la gestión y a las políticas culturales. En este trabajo se reflexiona sobre el estado de la cuestión en relación con la inteligencia artificial y con las actividades de los gestores culturales. Se aspira a plantear diferentes escenarios y, especialmente, a invitar a quienes tienen que tomar decisiones políticas y académicas a realizar propuestas ante los posibles futuros que ya hemos comenzado a vivir.
\end{abstract}

Palabras clave: industria cultural, inteligencia artificial, política cultural.

1 Entre la primera versión de estas páginas, de fines de noviembre de 2017, cuando constituyeron la conferencia que leí en el seminario Políticas y gestión cultural, en ocasión de la inauguración de la Cátedra Unesco en la Casa Rui Barbosa en Rio de Janeiro, y la presente, de febrero de 2018 para el número especial de Cuadernos del CLAEH, pasaron algunos eventos. El primero fue la acumulación exponencial de textos afines a lo aquí presentado; el segundo fue la lectura de Harari (2016) — como se verá luego-, a quien conocía de citas pero no había leído su Homo Deus, y el tercero, la decisión de organizar las Primeras Jornadas Inteligencia artificial y cultura para el 6 de abril de 2018 en el CuRE-Maldonado. 


\begin{abstract}
Given the inexorable progress and development of technological transformations and innovations, it seems that the time has come to ask ourselves if we are getting ready to face the challenges posed in terms of management and cultural policies. In this work, we reflect on the state of the art artificial intelligence and the activities of cultural managers. We aspire to put forward different scenarios and, especially, to invite those who have to make political and academic decisions to submit proposals for the possible futures that we have already begun to live.
\end{abstract}

Keywords: cultural industry, artificial intelligence, cultural policy.

Como bomberos en un mundo sin fuego, en el siglo XXI la humanidad necesita plantearse una pregunta sin precedentes: ¿qué vamos a hacer con nosotros? En un mundo saludable, próspero y armonioso, ¿qué exigirá nuestra atención y nuestro ingenio? Esta pregunta se torna doblemente urgente dados los inmensos nuevos poderes que la biotecnología y la tecnología de la información nos proporcionan. ¿Qué haremos con todo ese poder? Antes de dar respuesta a esta pregunta, necesitamos decir algunas palabras más sobre el hambre, la peste y la guerra. La afirmación de que los estamos poniendo bajo control puede parecer a muchos intolerable, extremadamente ingenua o quizá insensi-

ble. ¿Qué hay de los miles de millones de personas que consiguen apenas malvivir con menos de dos euros al día? ¿Qué pasa con la actual crisis del sida en África o las guerras que arrasan Siria e Irak? Para abordar estos problemas, dirijamos una mirada más detenida al mundo de principios del siglo XXI, antes de explorar la agenda humana de las

próximas décadas.

Homo Deus, Yuval Noah Harari

Las páginas que siguen probablemente se deban a mi obsesión por pensar tanto el pasado como el presente, pero sobre todo por tener conciencia de un futuro que se nos viene o ya está creciendo entre nosotros.

Frente a los muchos futuros posibles, creo que nuestra responsabilidad es tenerlos en cuenta de modo que emerja el mejor de los futuros posibles. No lo hago con un optimismo panglossiano, sino con el cuidado de no caer ni en la tentación de pensar que todo está bien en el mejor de los mundos posibles ni en la de pensar que todo futuro es inevitable. El o los futuros por construir son una responsabilidad que ningún ser humano puede evitar.

Por eso retomo la pregunta de Harari (2016): «¿Qué vamos a hacer con nosotros?», y la planteo en el marco de las políticas y la gestión cultural. Creo que el texto de Harari supera ampliamente el tema de este ensayo, pero también estoy convencido de que es 
un marco para pensarnos desde este hoy hacia un futuro que ya llegó. Y por eso la retomo, para que el tono de este ensayo no sea cuasi apocalíptico y aunque no sea escrito en el horizonte que Harari plantea de una lucha por la inmortalidad, la perspectiva de una sociedad crecientemente envejecida, como lo es la uruguaya, lo vuelve pertinente a nivel cultural.

\section{Introducción}

No pretendo revisar ni describir los avances en la investigación o en la implementación de la gestión y las políticas culturales en nuestra América. Esa es una tarea que, desde la Academia, se viene realizando de modo sostenido en las últimas décadas, y la multiplicación de licenciaturas, diplomas, maestrías y tesis demuestra que se va por buen camino.

Prefiero centrarme en los desafíos que, de un modo acuciante, la revolución tecnológica y la inteligencia artificial están comenzando a plantear, y lo harán en las próximas décadas respecto de la gestión y de las políticas culturales.

Ustedes dirán que me aparto del tema general de esta convocatoria. Puede ser, pero uno carga con sus obsesiones y con sus pasiones. No puedo reflexionar sobre la función de la gestión cultural sin pensar en que esta se lleva a cabo por seres humanos con formaciones, legados y valores diversos. Es cierto también que los gestores culturales operan de acuerdo a protocolos y saberes, pero aquí encuentro un desafío que siempre existió y ahora parece incrementarse.

Antes de ingresar en los desafíos que me obsesionan, debo explicitar desde dónde hablo. Lo hago, ya lo dije, desde mis obsesiones. La primera de esas obsesiones, que ha pautado gran parte de mis reflexiones - a lo largo de los años y en la práctica de implementar políticas culturales cuando me tocó la posibilidad de ejecutar mis ideas desde un cargo público-, es la de pensar en el otro, ese cualquiera del que algunos filósofos hablan, en relación con sus derechos culturales y con el ejercicio de su ciudadanía cultural.

Vengo pensando desde hace mucho en esto, pero últimamente me surgió una nueva preocupación: ¿es posible el cambio cuando toda sociedad carga con un legado o una inercia en relación con el papel de la cultura en la vida de la comunidad?

Creo que ningún gobierno, ningún Estado debe imponer una única visión de la cultura y mucho menos una única estética. Todos conocemos los efectos devastadores que distintos regímenes en Europa, en nuestra América y en los Estados Unidos - para remitirme solo al mundo más cercano a nuestras diversas herencias culturales - han tenido cuando propusieron nociones como arte degenerado, arte puro, cultura superior, realismo socialista y otras diversas formas de intolerancia, opresión y discriminación que se 
expresan, a veces, con una palabra o un calificativo como lo son pichi, cheto, plancha, cultura chatarra, culturoso, decadente y que recorren todos los registros del espectro ideológico y político de nuestra sociedad.

Podría recordar el impulso y la revisión que vivió la China de Mao cuando primero alentó la consigna «Dejad que cien flores florezcan, dejad que cien escuelas de pensamiento compitan ${ }^{1}$ y más tarde reprimió a quienes se habían atrevido a expresarse libremente hasta terminar en la despótica Revolución Cultural.

La consigna de dejar que cien flores florecieran puede haber sido una forma de plena libertad o una estratagema para obtener mayor control. No me interesa entrar en esto ahora, sino pensar en las contradicciones entre elaborar una política cultural democrática e inclusiva y los desafíos del poder hegemónico, así como en las tradiciones o inercias de las sociedades.

Todo esto viene a cuento de un intento por romper con el esquema de alta cultura versus baja cultura. Nuestras sociedades, si bien reconocen la heterogeneidad cultural existente entre nosotros, tienen una larga historia de instituciones e institucionalidades, al igual que gestiones culturales fundamentadas en el paradigma de una cultura patrimonialista y defensora de los grandes aportes de la alta cultura. La institucionalidad, y sobre todo la infraestructura cultural construida a lo largo del siglo XIX y la primera mitad del siglo XX, apostó — no estoy criticando sino describiendo- a los espacios museísticos, a los grandes teatros, a las orquestas nacionales y similares. La consigna era crear no solamente un aparato burocrático, legal y educativo, sino también estructuras e instituciones que permitieran armar el edificio cultural de nuestras naciones. Unas apostaron al modelo de las grandes colecciones y los grandes museos; otras, a la conservación patrimonial; otras, a la red de teatros y estímulos a la monumentalización urbana de ciudades, parques y todo aquello que consolidara el relato fundacional de la nación.

En ese proceso participaron no solo los Estados nacionales, sino también empresarios privados y, por supuesto, la Iglesia. Sin embargo, los emprendimiento de un sector de la sociedad - me refiero a los sectores marginales o subordinados- eran ignorados, censurados o, en el mejor de los casos, cooptados. La preocupación por el medio ambiente era escasa o se expresaba en la lógica de la colección de fauna y flora por medio de la creación de zoológicos y jardines botánicos.

¿Cuáles eran las posibilidades de introducir nuevos paradigmas de institucionalidad cultural frente a un legado determinante y hegemónico en nuestras sociedades? ¿Cómo interactuaba ese legado con el reconocimiento de la diversidad cultural y la multiplicidad de comunidades interpretativas (Stanley Fish, 1980) acerca de la expresión

1 La frase fue pronunciada por Mao en un discurso de 1957. La cita completa es: «Dejad que cien flores florezcan y cien escuelas de pensamiento compitan; es la política para promover el progreso en las artes y en las ciencias y una floreciente cultura socialista en nuestra tierra». 
artístico-cultural? Ese fue el desafío que nuevos movimientos sociales y nuevos acuerdos internacionales asumieron a partir de fines de la década de los setenta, en el último tercio del siglo XX, aun cuando este proceso se podría datar un par de décadas antes, con los acuerdos y las declaraciones impulsadas por UnEsCO.

Precisamente, desde el final del siglo xx y el comienzo del xxI se ha venido intentando - y no me parece mal- combinar lo uno y lo otro. Es decir, tratar de lidiar con paradigmas heredados, nuevas realidades sociales y nuevos acuerdos o declaraciones de alcance internacional. Encontramos ejemplos de estas nuevas realidades no solo en Uruguay sino también en Brasil, en Bolivia y en otros países de América Latina. Pero ese no es el problema.

La cuestión es otra; una cuestión que radica en la lucha y en las transformaciones que al legado hegemónico se le planteó al intentar conciliar lo heredado con lo emergente. Una solución consistió en ampliar, siempre dentro del paradigma tradicional, la inclusión de expresiones e instituciones culturales que incluyeran lo hasta entonces postergado o invisibilizado. Pero esto, que de hecho no era más que una salida en cierto sentido democratizadora, no cambiaba los paradigmas dominantes. Se trataba de un reconocimiento - en el sentido que Nancy Fraser (2001) le ha dado a este concepto-, pero no de un auténtico cambio.

La combinación entre lo heredado y lo emergente no supuso una transformación absoluta del paradigma inercial. ¿Estuvo mal? Quizás. ¿Era posible un cambio más radical al menos desde el Estado? No lo sé o no estoy seguro.

Creo, sin embargo, que el surgimiento de conceptos como los de diversidad cultural, derechos culturales y ciudadanía cultural constituyeron un punto de inflexión fundamental. En cierto sentido, tanto o más grande que el que supusieron las transformaciones de la cultura de masas o de las industrias culturales. Porque la lógica era/es otra.

Ejemplos en Uruguay -durante los últimos años- como los de las usinas culturales, los centros MEC y el Centro Cultural Urbano —este último para ciudadanos en situación de calle y que acoge, además, personas trans junto con una amplia variedad de población vulnerable - apuntan a consolidar la ciudadanía cultural y a reconocer que toda persona tiene derechos culturales. Algunos de estos casos han sido asimilados a los pontos da cultura de Brasil, aunque no sean exactamente lo mismo.

Este tipo de política cultural apunta, ya lo dijimos, a un cambio de paradigma. Sin embargo, su implementación parte de lógicas de gestión cultural diferentes y con planteamientos que hasta el momento no han podido ser conciliados, a pesar de haberse firmado acuerdos entre los dos países (Brasil y Uruguay) para estudiar la posibilidad de potenciar ambos emprendimientos.

Esto es y era así. Creo que se buscaba descentrar la lógica institucional de entidades que aspiraban a satisfacer exclusivamente las necesidades de los sectores medios de la sociedad. El descentramiento consistía en proponer la universalidad del 
ejercicio de los derechos culturales y evitar prácticas culturales en las que los ciudadanos eran sujetos pasivos consumidores de prácticas y eventos culturales, incluyendo la función de productores de cultura junto con la de meros consumidores.

Hasta aquí lo hecho, lo que se ha intentado, lo que se continúa realizando. Pero ¿cómo pensar el futuro ante los cambios sociales y tecnológicos que estamos viviendo y que seguramente vamos a vivir en décadas por venir? Hay escenarios futuros que en parte ya son realidades de este presente inquietante.

\section{Preguntas claras}

Apunto a la necesidad de pensar tanto en las políticas culturales como en los modelos de gestión culturales que se nos vienen y estamos empezando a vivir, que exigen preguntas a las que no hemos prestado mayor atención.

Hagamos esas preguntas:

1. La inteligencia artificial y la consecuente robotización ¿alcanzarán a los gestores culturales?

2. ¿Qué políticas culturales se implementarán en un escenario en el que las innovaciones tecnológicas de hoy se habrán vuelto obsoletas en los próximos veinte años? ¿El desempleo o la reconversión laboral aumentarán el tiempo libre o serán modos de exclusión que demandarán nuevas prácticas culturales, nuevos modelos de gestión cultural, nuevas políticas culturales?

3. ¿Qué pasará con la institucionalidad cultural tradicional, así como con las surgidas en los últimos años?

Se podrá argumentar que ese puente habrá que cruzarlo cuando lleguemos; sin embargo, creo que no pensar el, más que posible, muy probable escenario del futuro próximo es una muy mala decisión en política social, económica y cultural. Esto, seamos claros, independientemente de quiénes gobiernen en el futuro; hay cortes o napas culturales que atraviesan a todos los sectores políticos por igual.

Hay decisiones de larga duración que se deberían tomar antes de tratar los desafíos: ¿Qué tipo de sociedad queremos o aspiramos a defender hacia el futuro? ¿Debemos preservar lo que tenemos y a la vez estar abiertos a lo que se nos viene?

En algunos países se han implementado políticas públicas que — de acuerdo con las declaraciones internacionales sobre diversidad cultural, sobre derechos culturales y sobre la aspiración de una mayor equidad- intentaron salir de la trampa - heredada de varios siglos - de confundir políticas culturales con imposición de una 
cultura elitista que apostaba a la alta cultura y al patrimonio de lo venerado como monumento de la gran tradición. Esto se ha ido trasformando no solo por nociones patrimoniales que incorporaron el valor de lo intangible y de lo popular, sino también por el reconocimiento del valor cultural de las diversas comunidades, sin que ello implicara el olvido o el descarte de los grandes hitos de la llamada alta cultura.

¿Es posible, en el siglo XxI y en lo que se viene por delante, evitar estas transformaciones? Sí, claro, siempre es posible la quema de libros, la abolición de las conquistas sociales, educativas y culturales. Siempre es posible pensar en el surgimiento de movimientos sociales que propicien la censura y la represión de derechos adquiridos. Las restauraciones de Estados autoritarios son ejemplos que todos conocemos a lo largo de la historia - y me atrevo a decir en el presente- en nuestra América y en otras regiones del planeta. Pero Walter Benjamin (trad. 2009) tiene razón en afirmar:

Quien hasta el día actual se haya llevado la victoria, marcha en el cortejo triunfal en el que los dominadores de hoy pasan sobre los que también hoy yacen en tierra. Como suele ser costumbre, en el cortejo triunfal llevan consigo el botín. Se lo designa como bienes de cultura.

Esta visión de las Tesis sobre la filosofía de la historia expresa la posibilidad de un futuro vencedor en el que el botín de los bienes culturales sea apropiado por el conquistador de turno. Sin embargo, Benjamin (trad. 2009) está pensando en la usurpación del botín cultural, no en su destrucción. Está pensando o previendo el botín nazi del gran arte europeo, así como seguramente también en lo que ocurrió con todas las invasiones a lo largo de los siglos, que terminaron poblando los museos de muchos países de Occidente.

Me estoy refiriendo a otro aspecto, que también es parte de los bienes culturales, pero no a la materialidad patrimonial, sino a ese intangible que encarnan los derechos culturales y el concepto de la ciudadanía cultural, así como a prácticas y producciones culturales de los pueblos y no solo a lo realizado por las élites de los Estados que fueron devastados.

La cuestión es si una sociedad dominada por las learning machines y por la inteligencia artificial puede llegar a plantearnos una pérdida de esos derechos. El tema o la línea argumental que vengo construyendo tiene que ver con el presente debate entre lo que sería una visión utópica y otra distópica acerca de los acelerados avances de la revolución tecnológica en relación con la gestión y las políticas culturales. Tiene que ver además, entre los múltiples trabajos y prácticas que se augura van a ser realizados por seres informatizados o robots, con la posibilidad de que vayan a desaparecer los gestores culturales. 


\section{Escenarios posibles/probables}

Las preguntas o las posibilidades implícitas en la línea de argumentación que vengo desarrollando son múltiples. Abren la puerta a escenarios posibles no siempre probables, pero - y esto es lo que me importa - también a escenarios altamente probables.

El debate está instalado y tiene varios voceros: Mark Zuckerberg, Stephen Hawking, Lord Martin Rees, Ray Kurzweil, Elon Musk y Bill Gates, entre muchos otros científicos, empresarios, filósofos y pensadores que vienen considerando, promocionando o advirtiendo acerca de los futuros posibles, en ciertos casos, desde hace casi dos décadas (Castro, 2017). Algunos ven en las machines learning y en la inteligencia artificial un inexorable y deseado mundo feliz. Otros asimilan ese futuro a una reedición de la extinción de los dinosaurios, pero que esta vez alcanza la totalidad de la existencia humana. Basta leer el sitio Exponential-Technology ${ }^{2}$ para ver la variedad de escenarios con los que múltiples científicos ven el futuro cercano y lejano.

Nicholas Agar (2015), por ejemplo, sostiene, en una visión no distópica ni ludita, que en una economía sociodigital no todo será catastrófico:

No estamos en una buena posición para imaginar todos los trabajos de una economía sociodigital. Nuestras necesidades sociales son complejas. Habrá trabajos como el de los docentes, nurses, camareros, consejeros, actores, escritores (los pronósticos meteorológicos escritos por una computadora son buenos, pero a menudo cuando leemos deseamos estar en contacto con otro ser humano) y otros trabajos centrados en las habilidades de la gente. Las tecnologías van a reformular radicalmente nuestros papeles (o funciones). ${ }^{3}$

Agar representa una suerte de posición intermedia, ni totalmente utópica ni claramente distópica; en especial por el argumento de la necesidad de los seres humanos de interactuar afectivamente con otros seres humanos —al menos la mayoría-, algo que los robots u otros productos de la inteligencia artificial no podrían brindar. ${ }^{4}$

En una serie de conferencias en La Jolla, Lord Martin Rees (2016) tituló la tercera de sus presentaciones respecto de un futuro que fechó en el 2050 como The Dark Side

2 En https://www.huffingtonpost.com/topic/exponential-technology.

3 «We are not in a good position to imagine all the jobs of the social digital economy. Our social needs are complex. There should be jobs like teacher, nurse, waiter, counselor, actor, writer (computer-written weather forecasts are fine but most often when we read we want to be placed into contact with another human being) and other jobs centered on people skills. Technologies will radically reshape these roles».

4 Aunque hay casos en algunos países - Japón, por ejemplo- en los cuales la «afectividad» y la «eficiencia» de ciertas versiones actuales de robots no solo suplantan a camareros, sino también a las «parejas» afectivas. 
of World-Changing Technologies. En este lado oscuro, una visión claramente distópica. Entre sus muchas observaciones y evaluaciones, Rees (2016) se pregunta:

[¿Qué pasaría si] una máquina desarrollara una mente propia? ¿Permanecería siendo dócil? ¿Se volvería un ser errático o totalmente independiente ${ }^{5} \mathrm{Y}$ si pudiera infiltrar Internet - y la Internet de las cosas— podría manipular el resto del mundo. Podría tener objetivos completamente opuestos a los deseos humanos o incluso tratar a los humanos como un estorbo. ${ }^{6}$

Esta visión distópica se opone a otras «más rosadas», según palabras de Rees (2016), lo que indica la intensidad de un debate abierto.

Sin embargo, bombardeados como estamos en estos días por noticias acerca de nuevos inventos o descubrimientos, no es frecuente encontrar especulaciones o discusiones respecto a las transformaciones en el ámbito cultural. No es que no las haya, pero tienen un tono - si me permiten esta calificación- diferente. Algo de esto se puede ver en el último boletín de ACORNS, la asociación internacional también conocida como IFACCA, que reúne instituciones, agentes, productores y grupos de gestores artístico-culturales.

Tenemos muy presente el mundo digital y de la información en los últimos tiempos. Hemos empezado a trabajar en nuestro próximo informe D'Art 2018 sobre la digitalización y su impacto en las agencias gubernamentales de apoyo a las artes y la cultura, y la información está emergiendo como una parte importante de nuestras reflexiones en torno a la reciente consulta realizada a nuestros miembros y a medida que vamos renovando la entrega de nuestros servicios.

Por supuesto, no estamos solos. Esta semana, el Consejo de las Artes australiano ha publicado Haciendo funcionar el arte: un estudio económico de los artistas profesionales en Australia, que explora cómo la transformación digital está creando oportunidades y desafíos para los artistas, entre otras cosas. El informe incorpora una serie de paneles interactivos que permiten a los lectores explorar los datos de manera autónoma, poniendo de relieve cómo las agencias y los decisores políticos pueden sacar el máximo partido de las oportunidades digitales. También disfrutamos leyendo algunos de los principales hallazgos del Estado de los datos en abierto 2017, un informe del repositorio de investigación virtual Figshare que examina las

$5 \quad$ Go rouge? es la frase de Rees que no estoy seguro de traducir adecuadamente.

6 "What if a machine developed a mind of its own? Would it stay docile? Go rogue? If it could infiltrate the Internet - and the Internet of Things - it could manipulate the rest of the world. It may have goals utterly orthogonal to human wishes - or even treat humans as an encumbrance». 
actitudes globales hacia los datos en abierto y descubre que cada vez más investigadores están preparando sus datos para compartirlos.

En Sudáfrica, al hilo de la celebración del Día Mundial UnESCo del Patrimonio Audiovisual a finales de octubre, esta semana el Departamento de Artes y Cultura publicará las grabaciones digitalizadas del proceso de Rivonia, con el objetivo de concientizar globalmente sobre diversos aspectos de la conservación del patrimonio audiovisual. En Tailandia, el Ministerio de Cultura publicó una serie de nuevas aplicaciones para incrementar el acceso a y el conocimiento del patrimonio cultural nacional; también en las Américas, autoridades de Canadá y México se reunieron para analizar su agenda bilateral y centrarse en cuatro áreas prioritarias, incluida la agenda digital. Mientras tanto en Inglaterra, English Heritage ha lanzado una nueva colección virtual este mes, en colaboración con Google Artes y Cultura. (International Federation of Art Councils and Culture Agencies [IFACCA], 2018)

En realidad, el informe de ACORNS (IFACCA, 2018) parece formar parte del deslumbramiento general por los avances de la tecnología. No es incorrecto. Por el contrario, es cierto que en múltiples aspectos - conservación, registro, información e incluso en algunas áreas de las artes o de la economía creativa - los avances tecnológicos ayudan y permiten realizaciones antes más complejas, más costosas e incluso casi impensables.

Pero mi pregunta central es otra: ¿Es posible que los decisores de políticas o los gestores culturales sean sustituidos por robots? Rees (2016) señala en una de sus conferencias que los oficios de plomeros y de jardineros son de los pocos que no correrían peligro de ser robotizados. Siempre es posible, siempre todo o casi todo es posible; probable es otra cosa.

En línea con la afirmación de Rees (2016), uno de los argumentos que se escuchan o se leen en estos días afirma que los trabajos creativos no corren peligro. El poeta, el músico o el pintor son creativos, al menos los buenos. Los políticos o aquellos que toman decisiones a todo nivel — desde el gestor de un pequeño emprendimiento cultural hasta los ministros o directores de cultura - tienen entre sus múltiples responsabilidades decidir en función de impactos sociales, económicos, culturales y, obviamente, políticos. En ese sentido, cumplen una actividad que no dudo en llamar creativa, pues toda creación supone elección, y la elección es algo que caracteriza no solo la labor del artista, sino también de aquellos que instrumentan o regulan las prácticas culturales - ancestrales o no- de su comunidad.

No tendríamos por qué preocuparnos: la tarea de los gestores y de los decisores en materia cultural constituiría una labor creativa, tan creativa como la de los jardineros y los plomeros en el ejemplo de Lord Rees (2016). No cabe ningún tipo de duda. Claro, hasta que nos enteramos de la experiencia realizada en la Universidad Complutense de Madrid por Pablo Gervás (Fanjul, 2017), un profesor de la Facultad de Informática, 
quien diseñó y puso en práctica a WASP (Wishful Automatic Spanish Poet), un poeta automático. Gervás afirma:

Para desarrollar un ingenio de este tipo tenemos que entender cómo la mente lee, porque es la destinataria del poema, pero no necesariamente entender cómo la mente escribe (citado por Fanjul, 2017).

La información sobre la labor de Gervás (citado por Fanjul, 2017) indica cómo funciona su poeta automático. Así, se sostiene:

Dentro de su tarea entra la investigación de modelos cognitivos, la memoria, en fin, el pensamiento. A WASP se le dan instrucciones: la métrica, la rima; se le ordena inspirarse en la obra de un poeta. Y WASP obedece y escribe. Como los humanos, con el tiempo la máquina va aprendiendo nuevas formas de hacer poesía, y distingue mejor si lo que ha producido es buena poesía o ripios y frases banales.

Lo inquietante, al menos para mí, es que bajo la influencia de Lorca, WASP, este nuevo poeta automático, tejió —es interesante que se use la metáfora de tejer para describir la escritura de un poema- los siguientes versos:

Yunques ahumados

sus muslos se me escapaban como

peces sorprendidos

la mitad llenos de alas.

El debate en curso no solo se refiere a la creación o a las decisiones. Existen experimentos y pruebas acerca del papel de los expertos en arte, música y literatura. En este sentido, hay máquinas o inteligencia artificial que han llegado a valorar la obra de Munch y otros grandes, pero que descartan a Ingres, Renoir o Rodin.

Ante todo esto, ¿deberíamos preparar programas o software que funcionaran como asistentes de los gestores culturales?, ¿debería ser parte de los diplomas, maestrías y otro tipo de formaciones técnicas y académicas el aprender a manejar seres producidos por la inteligencia artificial?

Quizás no. Después de todo, hay quienes sostienen que nunca nos podrán quitar o nunca deberemos depositar o delegar la valoración, el gusto, la decisión acerca de qué cultura pretendemos desarrollar, gestionar, alentar o financiar en nuestras sociedades.

Los escenarios posibles/probables son múltiples. Las decisiones que tenemos que tomar como académicos y ciudadanos también lo son. Pero fundamentalmente son decisiones que no debemos posponer. 


\section{Diálogos y cuestiones finales}

Entiendo que, en relación con la formación de gestores culturales y de responsables de implementar o estudiar las políticas culturales, debemos iniciar o promover diálogos con el mundo académico que tiene formación en inteligencia artificial. Con ese mundo $\mathrm{y}$, por supuesto, con aquellos campos del conocimiento que atienden las políticas sociales y del trabajo. ${ }^{7}$

Entiendo, además, que hay que reivindicar el carácter creativo de los gestores culturales. Su profesionalización debería ser pensada para un futuro en el cual ya hemos comenzado a vivir. Se podría insistir y volver a preguntar si el de gestor cultural es un trabajo creativo.

Sí y no; siempre depende del ser humano y también de la tarea. Pero hay un reto que va más allá de lo que cada ser humano es o puede ser, un reto que incluye también las tareas que un gestor cultural debería desempeñar.

¿Hasta dónde queremos o debemos delegar en los seres producidos por la inteligencia artificial?

¿Cuántos de nosotros y de los actuales académicos o de los actuales gestores culturales están/estamos familiarizados con el mundo tecnológico de la inteligencia artificial?

Estamos viviendo un cambio civilizatorio que no se restringe al escenario de las conquistas por la diversidad cultural o la agenda de los derechos culturales y sociales de las llamadas poblaciones minoritarias de la segunda mitad del siglo Xx. Género, etnia, raza, opciones sexuales y otras preocupaciones y luchas del siglo xx siguen vigentes, pero surgió una nueva variable. Una variable que se presenta como democratizadora e inclusiva. Una variable que supone un escenario donde todas las sociedades, todas las minorías serían integradas y ejercerían sus derechos ciudadanos y culturales.

Y sin embargo, no. Este cambio civilizatorio puede llegar a generar nuevas exclusiones, nuevas marginaciones y nuevas poblaciones vulnerables. La transformación de las condiciones del trabajo, las eventuales multitudes desempleadas, las nuevas brechas sociales y culturales constituyen un desafío inmenso.

Si no nos preparamos para el futuro que ya llegó, si no repensamos la función de la cultura - la que heredamos, la que estamos viviendo y la que todavía no terminamos de imaginar cómo será- y no repensamos la función de quienes gestionan, administran o deciden, el tsunami de este escenario de mutación civilizatoria nos transformará en dinosaurios.

A lo mejor ya los somos y no nos hemos dado cuenta. 
No quiero terminar en una nota apocalíptica. Creo en la capacidad de nuestras sociedades para lidiar con los cambios. Creo que detrás de programas, software y todas las formas de inteligencia artificial hay un ser humano, y que esto seguirá así hasta que el momento de singularity del que hablan varios científicos posibilite que una máquina cree otra máquina con independencia del ser humano que alguna vez estuvo en el origen. Eso sí, también creo que si no empezamos a lidiar con lo que está ocurriendo y va a ocurrir en las próximas décadas, por más capacidad de imaginar o de inventar, como decía el maestro Simón Rodríguez (2004) hace casi dos siglos, vamos a terminar errando. 0 mejor, dicho con sus palabras, «inventamos o erramos».

\section{Bibliografía}

AGAR, N. (10 de julio, 2015). "The qualities most valued in employees are changing and not how you think». Recuperado de https://www.huffingtonpost.com/nicholasagar/qualities-valued-employees-changing_b_8253518.html.

BENJAmin, W. (2009). Tesis sobre la filosofía de la historia y otros fragmentos. Rosario, Argentina Prohistoria Ediciones.

CASTRO, M. (10 de noviembre, 2010). «Inteligencia artificial y machine learning». El Observador. Recuperado de https://www.elobservador.com.uy/inteligencia-artificial-y-machine-learning-n1137981.

Fanjul, S. C. (19 de abril, 2017). «¿Puede una máquina pintar como Picasso?». El País. Recuperado de https://elpais.com/elpais/2017/03/27/talento_digital/ 1490615561_931227.html.

FiSH, S. (1980). Is there a text in this class? The authority of interpretive communities. Cambridge: Harvard University Press.

FRASER, N. (2001). «Redistribución, reconocimiento y participación: hacia un concepto integrado de la justicia». En Informe mundial sobre la cultura, 2000-2001: diversidad cultural, conflicto y pluralismo. París: UNESCO. Recuperado de http:// www.inmujeres.gub.uy/innovaportal/file/21647/1/10_fraser2001_concepto_ integrado_justicia.pdf.

HARARI, Y. N. (2016). Homo Deus: Breve historia del mañana. Barcelona: Debate.

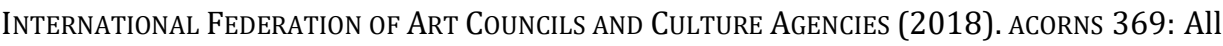
things data and digital. Recuperado de https://ifacca.org/en/what-wedo/knowledge-analysis/acorns/acorns-369-all-things-data-and-digital/

REES, M. (15 de febrero, 2016). «The dark side of world-changing technologies». Recuperado de https://www.huffingtonpost.com/martin-rees/world-changing-technologies_b_9236858.html.

RodríGuEZ, S. (2004). Inventamos o erramos. Caracas: Monte Avila Editores. 
TSE-TunG, M. (1960). Talks at the yenan forum on art and literature. $2^{\text {nd }}$. ed. Pekin: Foreign Languages Press.

TSE-Tung, M. (1965). Intervenciones en la Conferencia de Yenan sobre Arte y Literatura. Pekín: Ediciones en Lenguas Extranjeras. 